\title{
NEUERE TENDENZEN IM INTEGRATIONSRECHT LATEINAMERIKAS
}

\author{
Von Albrecht Weber
}

Nachdem sich der Integrationsprozeß in Lateinamerika in den letzten Jahren in einer anhaltenden, teilweise sogar akuten Krise befand, zeichnen sich doch in jüngster Zeit auch Innovationstendenzen ab, die eine resümierende Berichterstattung verdienen ${ }^{1}$. Die Gründe für die Krisis sind mannigfaltig und hier nicht näher darzulegen ${ }^{2}$; die Stagnation einzelner Integrationsverbände dürfte durch die inflationäre weltwirtschaftliche Entwicklung, die steigenden Zahlungsbilanzungleichgewichte und die schwankenden Rohstoff preise noch verstärkt worden sein ${ }^{3}$.

\section{Das Lateinamerikanische Wirtschaftssystem (SELA)}

Vor diesem Hintergrund ist als wichtigstes integrationspolitisches Faktum der letzten Jahre die Schaffung des "Lateinamerikanischen Wirtschaftssystems" (Sistema Economica Latinoamericana = SELA) am 17. Oktober 1975 in Pánama zu verzeichnen, dessen Gründungsvertrag - auch Pánama-Vertrag genannt - von 25 lateinamerikanischen Staaten unterzeichnet wurde und bereits am 7. Juni 1976 in Kraft trat ${ }^{4}$.

Die SELA ist eine internationale Organisation regionalen Zuschnitts mit Völkerrechtspersönlichkeit ${ }^{5}$. Zweck dieser erstmals nahezu alle Länder Lateinamerikas umfassenden Organisation ${ }^{6}$ sind die Förderung der intraregionalen Kooperation und die Schaffung eines Konsultations- und Koordinationssystems, um eine gemeinsame Haltung und Strategie in internationalen Gremien wie auch gegenüber Drittländern zu erreichen? ${ }^{7}$. Die gemeinsame Abstimmung nach außen nimmt einen wichtigen Stellenwert im Vertragswerk ein; damit erhalten die lateinamerikanischen Staaten innerhalb der „Gruppe der 77“, der Interessenvertretung der Entwicklungsländer, auch ein intraregionales Sprachrohr, das sich im Tauziehen um eine neue Weltwirtschaftsordnung bemerkbar machen dürfte. Die Präambel nimmt ausdrücklich auf die „Erklärung und das Aktionsprogramm für eine neue Weltwirtschaftsordnung" und die "Charta für wirtschaftliche Rechte und Pflichten" Bezug und zeichnet damit programmatisch die künftige "Außenpolitik“ vor ${ }^{8}$. Schwer-

$1 \mathrm{Vgl}$. die früheren Berichte von Hummer, Regionale und Subregionale Integrationszonen in Lateinamerika und der Karibik, VerfRU 9 (1976), S. 71 ff.; ferner Roberts- Newman, Latin American Integration, Lawyer of the Americas 8 (1976), S. $474 \mathrm{ff}$., die in ihrer Darstellung vor allem dem Karibischen Gemeinsamen Markt (CARICOM) breiten Raum widmen und der hier nicht näher zu behandeln ist.

2 Hinweise bei Petersmann, Wirtschaftsintegrationsrecht und Investitionsgesetzgebung der Entwicklungsländer (1974), S. $35 \mathrm{ff}$.; Hummer, Subregionale Präferenzzonen als Mittel lateinamerikanischer Integrationspolitik, Zeitschrift für Lateinamerika, Wien, 8 (1975), S. $119 \mathrm{ff}$.

$3 \mathrm{Vgl}$. die Sitzung der Exekutiven lateinamerikanischer Integrationseinrichtungen am 21./23. 10. 1976 im Rahmen der SELA, Integración Latinoamericana 1 (1976), Nr. 9, S. 47.

4 Text des Vertrags in ILM 15 (1976), S. $1081 \mathrm{ff}$. Eine Darstellung der SELA findet sich bei Alejo-Hurtado. EL SELA. Un mecanismo para la acción (1976).

5 Die Völkerrechtspersönlichkeit ergibt sich bereits aus einer ausdrücklichen Verleihung im Gründungsvertrag (Art. 2 SELA-V).

6 Nichtmitglieder sind bislang nur einige Inselstaaten der Karibik (Domenica, St. Kitts-Nevis-Anguilla, Sta. Lucia, S. Vicente, Montserrat, Antigua, Belice), für die aber der Beitritt gemäß Art. 7 SELA-V offen bleibt.

7 Abs. 1 der Präamel, Art. 2, 3 SELA-V. recht, ZaöRV 36 (1976), S. $492 \mathrm{ff}$.; Weber, Die Neue Wirtschaftsordnung: Die Ergebnisse der 7. Sondergeneralversammlung der Vereinten Nationen, RIW/AWD (1977), S. 21 ff. m.w.N. 
punkte der Positionsabstimmung sind die Verhandlungen im Rahmen der Unctad, Unido, der Konferenz für internationale wirtschaftliche Zusammenarbeit (NordSüd-Dialog), die Arbeiten an einem Verhaltenskodex für transnationale Unternehmen sowie der gemeinsame Stellungsbezug gegenüber den USA und der EG. Der Organisation dürfte in gewissem Umfang eine Außenvertretungsbefugnis im Rahmen internationaler Konferenzen eingeräumt sein ${ }^{10}$; eine Verhandlungs- oder Abschlußkompetenz der Organisation, die jene der Mitgliedstaaten in den angesprochenen Bereichen ausschlösse, läßt sich jedoch dem Vertragstext weder ausdrücklich noch stillschweigend entnehmen; es erscheint sogar fraglich, ob eine mit den Mitgliedstaaten konkurrierende Vertragsschlußkompetenz der SELA begründet ist ${ }^{11}$. Der Vertrag hebt als grundlegende Prinzipien der Organisationstätigkeit die „klassischen" völkerrechtlichen Grundrechte der Souveränität, Gleichheit, Unabhängigkeit und Nichteinmischung in die inneren Angelegenheiten hervor, die heute von den Entwicklungsländern den Industrienationen gerne bei der Diskussion um eine neue Wirtschaftsordnung entgegengehalten werden ${ }^{12}$; daneben findet sich auch das Prinzip der "Solidarität“, das - auf eine Materialisierung des Gleichheitssatzes hinauslaufend - einen der tragenden Pfeiler im Argumentationsrahmen der Entwicklungsländer bildet ${ }^{13}$. Vertraglich niedergelegte Ziele der SELA sind:

- Förderung der intraregionalen Zusammenarbeit der Staaten;

- Stärkung der regionalen und subregionalen Integrationsprozesse;

- Ausgestaltung und Durchführung von Wirtschafts- und Sozialprogrammen;

- vorzugsweise Behandlung der weniger entwickelten Länder;

— Unterstützung der Länder mit „begrenzten Märkten“ und der „landlocked states" 14 .

Aus diesem Zielkatalog ergibt sich, daß die SELA nur als Dachorganisation der lateinamerikanischen Staaten und der von diesen gebildeten Integrationsverbän$\operatorname{de}^{15} \mathrm{zu}$ verstehen ist. Die auf die lateinamerikanischen Integrationsverträge besonders treffende Kennzeichnung als „Rahmenverträge“ („tratado-marco“) mag auf die SELA in verstärktem Maße zutreffen ${ }^{16}$. Anfängliche Hoffnungen einiger Beobachter, die SELA könnte die bisherigen Integrationsprozesse ersetzen, erscheinen angesichts der bloßen Klammerfunktion und mangels weitreichender Kompetenzübertragung auf die neue Organisation unbegründet. Ihre integrative Funktion kann die SELA nicht durch Liberalisierung einzelner Wirtschaftssektoren

9 Decision No. 7 des Latin American Council vom 16. 6. 1987: First Work Program of SELA, ILM 15 (1976), S. $1125 \mathrm{ff}$. So hat die SELA bereits Kontake zur EWG aufgenommen, die sich am Arbeitsprogramm der SELA beteiligen will; Boselli, Die Beziehungen zwischen der Europäischen Gemeinschaft und Lateinamerika, EA (1977) Nr. 14, S. $427 \mathrm{ff}$.

$10 \mathrm{Vgl}$. etwa Art. 3, Ziff. 4 SELA-V. Die künftigen Außenvertretungsbefugnisse werden sich erst anhand der Praxis der Organisation ermitteln lassen.

11 Die Verhandlungs- und Abschlußbefugnis des Exekutivorgans der SELA, des „Permanent Secretariat“, nach Art. 31, Ziff. 8 SELA-V bezieht sich nur auf "arrangements “ mit internationalen Organisationen und Behörden, sowie Behörden von Mitglieds- und Drittstaaten, dürfte also die "technischen ${ }^{\alpha}$ Verbindungen, Absprachen und Vereinbarungen umfassen, die herkömmlicherweise von den Exekutivorganen internationaler Organisationen gepflegt werden. Vgl. etwa Art. 229-231 EWG-V oder Art. 12 OECDKonvention.

12 S. Präambel und Art. 4 SELA-V

13 Vgl. etwa Kemper, Nationale Verfügung über natürliche Ressourcen und die Neue Weltwirtschaftsordnung der Vereinten Nationen, Schriften zum Völkerrecht (1976), S. $56 \mathrm{ff}$.

14 Art. 5, Ziff. 1, 2, 3, 5 SELA-V.

15 Lateinamerikanische Freihandelszone = ALALC; Andenpakt = PA; Zentralamerikanischer Gemeinsamer Markt = MCCA; Karibischer Gemeinsamer Markt = CARICOM; Ostkaribischer Gemeinsamer Markt = ECCM; subregionale Ưbereinkunft zur Koordinierung der. Wirtschaftspolitiken Uruguays, Paraguays, Boliviens = URUPABOL; Subregionale Ubereinkunft zur infrastrukturellen Entwicklung des La-PlataBeckens = Cuenca del Plata; s. hierzu die in Anm. 1 und 2 angegebene Literatur.

16 Zum Begriff des Rahmenvertrags (traité-cadre) etwa Orrego-Vicuña, Contemporary International Law in the Economic Integration of Latin America. Problems and Perspectives in: Legal Aspects of Economic Integration, Haager Kolloquium (1971), S. $106 \mathrm{ff}$; Intal, Derecho de la Integración Latinoamericana (1969), S. $1037 \mathrm{ff}$. 
erfüllen, da ihr in dieser Hinsicht keine Zuständigkeiten übertragen sind. Das vom Rat mittels Entscheidung vom 16. Juni 1976 verabschiedete erste Arbeitsprogramm $^{17}$ sieht als vorrangige Aufgaben die Kooperation im Landwirtschafts- und Industriesektor, die Errichtung eines Informationssystems („SILA“) wie eines Verbundsystems technologischer Informationen („RITLA“), die Zusammenarbeit auf dem Rohstoffsektor und im technischen Bereich vor.

Höchstes Organ der SELA ist der "Lateinamerikanische Rat" (Latin American Council), der sich aus je einem Vertreter eines Mitgliedstaates zusammensetzt und - als Staatenvertretungsorgan - den entsprechenden „Räten“ der lateinamerikanischen Integrationsverbände ähnelt ${ }^{18}$. Ihm steht unter anderem die Formulierung der allgemeinen politischen Leitlinien, die Budgetbewilligung und Festlegung der Mitgliedsquoten ${ }^{19}$, die Interpretationsbefugnis der Satzung, die interne Leitungsfunktion gegenüber den SELA-Organen, die Billigung der gemeinsamen externen Verhandlungsposition sowie eine allgemeine Implementierungs- und Entscheidungsbefugnis $^{20}$ zu. Der Rat tritt mindestens einmal jährlich auf ministerieller Ebene zu einer ordentlichen Sitzung zusammen. Er erläßt (an die Staaten gerichtete oder interne) Entscheidungen, wobei die wichtigeren einstimmig, alle übrigen mit $Z$ weidrittelmehrheit aller anwesenden oder einer einfachen Mehrheit aller Staaten zu fällen sind ${ }^{21}$. Das Mehrheitsprinzip erfährt aber dadurch eine gewisse Relativierung, als Entscheidungen nicht die nationale Politik in widriger Weise beeinträchtigen dürfen ${ }^{22}$, was letztlich wieder einen allgemeinen Konsens erfordern dürfte; Abkommen und Projekte regionaler Kooperation binden nur die jeweils teilnehmenden Staaten ${ }^{23}$. Eine wichtige Rolle in der Organstruktur der SELA nehmen die durch Entscheidung des Rates oder der interessierten Staaten zu errichtenden Aktionskomitees ("Action Committees") ein. Die Errichtung von Ausschüssen ist auch anderen lateinamerikanischen Organisationen geläufig und dient der funktionellen Implementierung der Organisationsziele ${ }^{24}$. Die aus Staatenvertretern zusammengesetzten Aktionskomitees werden nur für die Dauer ihrer spezifischen Aufgabenerfüllung tätig und dienen der Durchführung besonderer Studienprogramme sowie der Vorbereitung gemeinsamer Verhandlungspositionen ${ }^{25}$. Technisches Administrativ- und Exekutivorgan der SELA ist das "Permanente Sekretariat" in Cáracas (Art. 27 SELA-V), das die für internationale Organisationen typischen Verwaltungsaufgaben eines Sekretariats erbringt ${ }^{26}$. Insgesamt ist die SELA als ein

17 S. Anm. 9.

18 Art. 9-13 SELA-V; vgl. den Zentralamerikanischen Wirtschaftsrat (Art. XX Abs. 1 MCCA), die Kommission den Andenpakts (Art. 6 PA) oder den Rat der Außenminister der ALALC nach dem Protokoll vom 12. 12. 1966 (Anm. 118).

$19 \mathrm{Im}$ ersten Jahr betrug das Budget 1,9 Mio. US-Dollar, wooei die Quoten nach vier Ländergruppen festgelegt wurden; s. Roberts-Newman, a. a. O. (Anm. 1), S. 477.

$20 \mathrm{Vgl}$. Art. 15, Ziff. 16 und 17 SELA-V; diese Bestimmungen dürften sich als eine ausdrückliche "implied powers"-Klausel verstehen lassen, die der Organisation alle zur Durchführung ihrer Aufgaben notwendigen Befugnisse einräumen.

21 Die Formulierung der allgemeinen Politik, Interpretation der Satzung, Billigung von Vertragsänderungen und Verabschiedung einer externen Verhandlungsposition muß im Konsens erfolgen (Art. 17 a SELA-V).

22 Art. 19 SELA-V: "The Latin American Council shall not take decisions adversely affecting national policies of the Member States".

23 Art. 18 SELA-V.

24 Vgl. etwa die "Technische Kommission“, „Beigeordneten Räte“ und „Beigeordneten Kommissionen“ der ALALC.

25 Art. 20, 21 SELA-V. Die Bedeutung der Aktionskomitees läßt sich auch daran ablesen, daß bereits die Ratsentscheidung Nr. 5 die Verfahrensregeln der Komitees verabschiedete, SELA Document vom 16. 6. 1976, in: ILM 15 (1976), S. $1118 \mathrm{ff}$. So wurden etwa Aktionskomitees eingerichtet für Getreide, Samen, Obst; für Meeresfrüchte; für Fleisch- und Milchprodukte, s. Integración Latinoamericana (1977), Samen, Obst; für Meeresfrüchte; für Fleisch- und Milchprodukte, s. Integración Latinoamericana (1977),

$26 \mathrm{Vr}$. 13, S. 62. des Andenpakts nimmt zugleich Sekretariatsaufgaben wahr (Art. 15 [i] PA); das CARICOM-Sekretariat (Art. 15 CARICOM-V); das Ständige Sekretariat des MCCA = SIECA (Art. XXIV MCCA-V). 
hoffnungsvoller Schritt zu einer stärkeren Koordinierung der vielfältigen Integrationsbemühungen in Lateinamerika $\mathrm{zu}$ werten ${ }^{27}$, auch wenn sie keinen unmittelbaren Ansatz zu dem erstrebten Lateinamerikanischen Gemeinsamen Markt bietet, wie es Art. 54 ALALC-V vorsah und die „Deklaration der Präsidenten von Amerika" in Punta del Este am 14. 4. 1967 stipulierte ${ }^{28}$.

2. Der Zentralamerikanische Gemeinsame Markt (MCCA) und das Vertragsprojekt der Wirtschafts- und Sozialgemeinschaft Zentralamerikas (CESCA)

Erneuerungstendenzen, die bereits bis zur Vorlage eines neuen Vertragswerks gediehen sind, lassen sich auch für den Bereich der mittelamerikanischen Wirtschaftsintegration feststellen. Der Zentralamerikanische Gemeinsame Markt (MCCA), 1960 zwischen El Salvador, Guatemala, Honduras und Nicaragua begründet und 1963 um den Beitritt Costa Ricas erweitert ${ }^{29}$, stellt das Kernstück eines Netzes von Integrationsverträgen der zentralamerikanischen Staaten dar ${ }^{30}$. Der MCCA galt als einer der vielversprechendsten Integrationsversuche Lateinamerikas, bis der zwischen Honduras und El Salvador 1969 ausgelöste „Fußballkrieg" seine Grundlagen erschütterte und als dessen Folge Honduras seinen Markt zeitweilig ausgliederte ${ }^{31}$. Den negativen Auswirkungen dieser schweren Krise versuchten die Staaten des "Gemeinsamen Marktes“ 1972 durch die Einsetzung eines „Hohen Komitees für die Ausgestaltung und Umstrukturierung des Gemeinsamen Marktes"32 Einhalt zu gebieten, das die Möglichkeiten einer Neugestaltung des Marktes ergründen sollte ${ }^{33}$. Nach zweieinhalbjähriger Arbeit legte das Komitee am 23. März 1976 den Präsidenten der zentralamerikanischen Republiken das „Projekt eines Vertrags der Zentralamerikanischen Wirtschafts- und Sozialgemeinschaft" vor ${ }^{34}$, das inzwischen den staatlichen Stellen der Mitglieder zur Prüfung vorliegt. Wenn auch eine Unterzeichnung des Vertrags in der vorgelegten Fassung angesichts gewichtiger Einwände der Mitgliedstaaten noch fraglich erscheint, dürfte doch nunmehr eine grundsätzliche Ablehnung von seiten Honduras' ausscheiden, da der Bruderzwist zwischen Honduras und El Salvador durch eine Vereinbarung über friedliche Streitbeilegung und Normalisierung der gegenseitigen Beziehungen ausgeräumt sein dürfte ${ }^{35}$.

Das Vertragsprojekt versucht den Empfehlungen des Mandats der zentralamerikanischen Präsidenten gerecht zu werden, indem es in pragmatischer Weise politische

27 Vgl. Mayorga - Cortés (Generalsekretär der SIECA), Mercado Común Centroamericano: Situación actual y perspectivas, in: Integración Latinoamericana 1 (1976), Nr. 8, S. $12 \mathrm{ff}$.

28 Erklärung I, a, b; EA (1967) D 513 ff. Dieser Markt soll durch einen Zusammenschluß von ALALC und MCCA erfolgen, eine angesichts der gegenwärtigen wirtschaftlichen Realitäten eher ferne Zukunftsperspektive.

29 "General Treaty of Central American Economic Integration ${ }^{\alpha}$, abgedruckt in Intal, Instruments of "Economic Integration in Latin America and the Caribbean, Bd. II, 1975, S. 385 ff.; deutsche Ubersetzung bei Schulz, Wirtschaftliche Integration in Mittelamerika. Rechtlicher Rahmen und sozioökonomische Wirklichkeit, Diss. Münster (1973), S. $232 \mathrm{ff}$. Der Vertrag trat am 4. 6. 1961 in Kraft.

30 Eine Aufzählung der bis 1972 abgeschlossenen Abkommen findet sich bei Schulz, ebd., S. $274 \mathrm{ff}$

31 Nachweise bei Schulz, ebd., S. 131 ff.; Mayorga-Cortés will sogar drei Krisenphasen (1966-68; 1969-72; 1974-76) unterscheiden, a. a. O. (Anm. 27), S. 15.

32 "Comité de Alto Nivel para el Perfeccionamiento y la Reestructuración del Mercado Común Centroamericano ${ }^{\alpha}=$ CAN.

33 „Centroamerica: Reestructuración del Mercado Común Centroamericano. Comité de Alto Nivel“, in: Derecho de la integración (1974) Nr. 14, S. $242 \mathrm{ff}$.

34 "Informe del Comité de Alto Nivel para el Perfeccionamiento y la Reestructuración del Mercado Común Centroamericano presentado a los señores presidentes de las republicas centroamericanas ${ }^{*}$, in: Integración Latinoamericana (1976) Nr. 5, S. 52 ff.; Vertragstext daselbst S. 59 ff. und Nr. 6, S. 63 ff.; Nr. 7, S. 67 ff.; Nr. 8, S. 50 ff.

35 Übereinkunft vom 6. 10. 1976, Integración Latinoamericana Nr. 8 (1976), S. 41. 
und technische Durchführbarkeit mit angemessener Beteiligung aller fünf zentralamerikanischen Staaten am Integrationsprozeß und fortschreitender Integrationsverflechtung $\mathrm{zu}$ vereinen sucht. Eine detaillierte Darstellung dieses 311 Artikel zählenden Vertragsentwurfs ist schon aus räumlichen Gründen nicht möglich, erscheint aber auch insoweit verzichtbar, als der Entwurf angesichts der mitgliedstaatlichen Einwände noch einige Korrekturen erfahren dürfte ${ }^{36}$. Gleichwohl erscheint ein Vergleich dieses Vertragswerks mit dem bisher erreichten Integrationsstand interessant.

Während der MCCA sein Ziel in der Errichtung einer Freihandelszone und der Einführung eines gemeinsamen Außenzolls sah ${ }^{37}$, will die CESCA das Erreichte nicht nur erhalten, sondern durch einen umfassenderen Integrationsansatz den wirtschaftlichen Entwicklungsprozeß dynamisieren. $\mathrm{Zu}$ diesem Zwecke strebt der Vertrag nicht nur die Ausgestaltung der Freihandelszone ${ }^{38}$ und die Harmonisierung der Ursprungs-, Wettbewerbs- und Steuerregelungen an (Art. 89-124 CESCA-V), sondern sucht auch die Zollunion auszubauen (Art. 125-136 CESCA-V), eine Währungsunion $z u$ errichten (Art. 137-143 CESCA-V) und die freie Mobilität der Produktionsfaktoren sicherzustellen (Art. 144-161 CESCA-V). Die Uberschrift dieses dritten Teils des Vertrags („Mercado Comun“ = Gemeinsamer Markt), der die eben genannten Zielsetzungen umfaßt, ließe zunächst darauf schließen, daß sich das mittelamerikanische Integrationsverständnis dem europäischen insoweit angenähert habe, als unter "Gemeinsamer Markt" die Liberalisierung der Binnen- und Außenzölle und die freie Zirkulation der Produktionsfaktoren zu begreifen sei, ${ }^{39}$ doch steht dem Titel I entgegen, der unter der Bezeichnung "Perfeccionamiento del Mercado Comun“ (- Vollendung des Gemeinsamen Marktes) nur die Normen über freien Warenverkehr, Wettbewerb, Steuer- und Zollangleichung enthält. Daraus wird ersichtlich, daß die mittelamerikanische Integrationsterminologie noch keine klaren Konturen gewonnen hat und auch künftig nicht mit dem anerkannten Integrationsverständnis westlicher Autoren gleichzusetzen ist ${ }^{40}$. Die Angleichung der Binnen- und Außenzölle war bereits aufgrund der bestehenden Integrationsverträge weit gediehen ${ }^{41}$. Der Vertragsentwurf sieht nunmehr die etappenweise Errichtung der vollständigen Zollunion aufgrund von Programmen vor, die den Koordinationsgrad der Volkswirtschaften im monetären, fiskalischen, agrarischen, industriellen, wechselkurs- und zollpolitischen Sektor ausreichend berücksichtigen. Die Leitprinzipien der stufenweisen und progressiven Integration ("gradualidad y progresividad“) ${ }^{42}$ werden auch aus der Flexibilität ersichtlich, mit der der Rat - auf Vorschlag der Kommission - die Zollstufen festsetzen kann; der Vertrag schreibt diesbezüglich keine Fristen vor ${ }^{43}$, was in der

\footnotetext{
36 Vgl. Mayorga-Cortés, a. a. O. (Anm. 27), S. 20.

37 Hierzu näher Peterwerth, Das Vertragswerk des Zentralamerikanischen Gemeinsamen Markts (1973), S. $19 \mathrm{ff}$.

38 Vgl. Art. 89 I Cesca-V.

39 Der EWG-V enthält keine Definition des „Gemeinsamen Markts“, doch lassen sich Art. 2, 3 EWG-V die genannten Elemente entnehmen, s. Ipsen, Europäisches Gemeinschaftsrecht (1972), S. $545 \mathrm{ff}$.

40 Zum herrschenden Begriffsverständnis Bela Balassa, Towards a Theory of Economic Integration, in: Kyklos, Vol. XIV (1961), S. 5; zum lateinamerikanischen Verständnis Alemán, Los Conceptos JurídicoEconomicos de Preferencia, Zona de Libre Comercio, Unión Aduanera, Asociación Economica, Comunidad Economica, Mercado Común y Unión Economica, in: Anuario Uruguayo de Derecho Internacional IV $(1965 / 66)$, S. 35.

41 Vgl. etwa die Angleichung des gemeinsamen Außenzolls aufgrund des Zentralamerikanischen Abkommens über die Angleichung der Importzölle vom 1. 9. 1959. So konnten 97,5 Prozent aller Warenpositionen vereinheitlicht werden, s. Peterwerth, a. a. O. (Anm. 37), S. 56.

42 Art. 128 CESCA-V.

43 Art. 129 CESCA-V.
} 
lateinamerikanischen Integration einmalig ist. Diese Vorsicht läßt sich nur mit den negativen Erfahrungen der mittelamerikanischen Integration erklären, die die vorgegebenen Fristen nicht einhalten konnte. Die vorhandenen Ansätze der bereits im MCCA-V angestrebten Währungsunion ${ }^{44}$, die $\mathrm{zu}$ einer zentralamerikanischen Clearing-Stelle ${ }^{45}$ und einem „Abkommen zur Schaffung einer zentralamerikanischen Währungsunion" 46 geführt hatten, werden ausgebaut. Fernziel der Währungsunion, für die in weiser Voraussicht ebenfalls kein Termin gesetzt ist ${ }^{47}$, ist die Schaffung einer zentralamerikanischen Währungszone mit einem gemeinsamen Zahlungsmittel ${ }^{48}$. Als Recheneinheit im Zentralbankverkehr dient der „Peso Centroamericano" (\$C. A.), der bereits im Rahmen des zentralamerikanischen Clearing-Systems eine Rolle spielte ${ }^{49}$. Das im IWF-Abkommen festgelegte Paritätensystem ist Referenzpunkt ${ }^{50}$ dieser Recheneinheit, die aber nach Inkrafttreten der zweiten Satzungsnovelle des IWF eine entsprechende Orientierung an der seit 1. Juli 1974 geltenden Standardkorbmethode erfahren dürfte ${ }^{51}$. Zum Zwecke der progressiven Errichtung der Währungsunion verpflichtet der Vertrag nicht nur zur gegenseitigen Konvertibilität der Währungen, sondern auch zur Freiheit des Zahlungsverkehrs ${ }^{52}$. Als wesentlichen Fortschritt der mittelamerikanischen Integrationsbemühungen läßt sich die angestrebte Liberalisierung des Personen-, Dienstleistungs- und Kapitalverkehrs bezeichnen ${ }^{53}$; damit wären die vier Grundfreiheiten des EWG-Vertrags (freier Waren-, Personen-, Dienstleistungs- und Kapitalbzw. Zahlungsverkehr) in ähnlicher Weise garantiert. Der Vertrag sieht dementsprechend die Freizügigkeit aller Staatsangehörigen (Art. 148 CESCA-V), unter besonderer Berücksichtigung der Freizügigkeit der Arbeitnehmer (Art. 150-155 CESCA-V) sowie die Niederlassungsfreiheit der selbständig Erwerbstätigen und Unternehmer (Art. 156 CESCA-V) vor. Die Normierung der Liberalisierungs.pflichten des freien Personen-, Dienstleistungs- und Kapitalverkehrs ist weit unbestimmter als im EWG-Vertrag gefaßt ${ }^{54}$ und überläßt die nähere Ausgestaltung jeweils dem "Rat" auf Vorschlag der „Kommission"55, so daß auch hier die Kennzeichnung als „Rahmenvertrag“ angebracht erscheint ${ }^{56}$. Inwieweit die Liberalisierungsziele von der künftigen CESCA erreicht werden, wird von der pragmatischen und flexiblen Handhabung der zugewiesenen Organbefugnisse und dem Verzicht auf eine souveränitätsorientierte Vertragsauslegung abhängen; die entsprechenden „ordre public“-Vorbehalte des Vertrags scheinen bedenklich weit

\footnotetext{
44 Art. X MCCA-V.

45 Agreement Establishing the Central American Clearing House vom 28. 7. 1961, Intal, Instruments, II, a. a. O. (Anm. 29), S. $515 \mathrm{ff}$.

46 Agreement on the Establishment of a Central American Monetary Union vom 25. 2. 1964, ebd., S. $523 \mathrm{ff}$. Dieses Abkommen beschränkt sich im wesentlichen auf ein Informations- und Konsultationssystem sowie ein gemeinsames Wechselkurssystem der Zentralbanken.

$47 \mathrm{Vgl}$. auch Informe, a. a. O. (Anm. 34), S. 54.

48 Art. 137, 138 CESCA-V.

49 S. Art. 5 des Clearingabkommens (Anm. 45).

$50 \mathrm{Vgl}$. Art. 140 Abs. 2 CESCA-V: „... el valor del Peso Centroamericano con base en el sistema de paridades monetarias legales previsto en el Convenio del Fondo Monetario Internacional“.

$51 \mathrm{Vgl}$. Weber, Die 2. Satzungsnovelle des Internationalen Währungsfonds und das Völkerrecht, Festschrift für F. A. Mann (1977), S. $807 \mathrm{ff}$.

52 Art. 139 CESCA-V.

53 Titel IV ("Circulación de Factores“): Art. 144-161 CESCA-V. Eine ähnliche Verpflichtung - allerdings in allgemeinerer Form - sah auch Art. I, II des "Vertrags über die wirtschaftliche Assoziation ${ }^{\alpha}$ von Guatemala am 6. 2. 1960 zwischen El Salvador, Honduras und Guatemala vor.

54 Einen Vergleich der Liberalisierung des Personenverkehrs im EWG-V und im CESCA-V enthält die Studie der SIECA: "La Circulación de Personas en el Proyecto de Tratado de la Comunidad Economica y Social Centroamericana ", Cuadernos de la Sieca, No. 2 (1976).

55 Vgl. Art. 146, 148 Abs. 2, 150, 153, 159, 161 Abs. 3 CESCA-V. Dies gilt insbesondere für die Nichtfestlegung von Fristen und die weiten Handlungsspielräume von Rat und Kommission.

56 So auch die SIECA-Studie: Principales Aspectos Jurídico-Institucionales del Proyecto de Tratado de la Comunidad Economica y Social Centroamericano, Cuadernos de la Sieca, No. 1, 1976, S. $11 \mathrm{ff}$.
} 
gefaßt ${ }^{57}$. Während die Koordinierung und Förderung der zentralamerikanischen Industrien schon bisher einen wichtigen Stellenwert in der Integrationsverpflichtung einnahm ${ }^{58}$, sollen nun die Grundstoffindustrien durch eine gemeinsame Planung und andere Industriesektoren durch Abschluß von Vereinbarungen eine Förderung erfahren ${ }^{59}$. Der ambitionierte Intregrationsansatz des CESCA-Entwurfs läßt sich auch an der Zielvorgabe einer ausgewogenen wirtschaftlichen Entwicklung aller Mitglieder sowie einer gemeinen Sozial- und Agrarpolitik ablesen, die bislang nicht zum zentralamerikanischen Integrationshorizont gehörten ${ }^{60}$. Zum Zwecke einer ausgewogenen Wirtschaftsentwicklung zwischen Ländern, Regionen oder Tätigkeitsbereichen kann die Gemeinschaft alle hierzu erforderlichen wirtschaftspolitischen Maßnahmen ergreifen, so durch Industrie- und Agrarplanung, Errichtung von Spezialfonds, Zollpolitik und Ausnahmeregelungen vom Freihandel (Art. 167 CESCA-V). Die Sozialpolitik ist durch Sicherung der Arbeitsplätze und gerechte Entlohnung, Koalitionsfreiheit und Tarifautonomie, Sozialversicherung und Gesundheitsplanung, Ernährungs- und Wohnungsbauprogramme und die Harmonisierung der Erziehungssysteme zu verwirklichen (Art. 170-197 CESCA-V). Vorrangiges Ziel der gemeinsamen Agrarpolitik ist die Steigerung der landwirtschaftlichen Produktion zur Deckung des heimischen Verbrauchs, Diversifizierung und Erhöhung der Exporte und Exportstubstitution und allgemein die Verbesserung der Lebensverhältnisse der Bevölkerung (Art. 198 CESCA-V). Der Entwurf sieht Normen über regionale Landwirtschaftsprogramme, Finanzierung, Kommerzialisierung und Stimulierung der Agrarentwicklung vor und enthält - wie auch in anderen Bereichen - eigene Schutzklauseln für ernste Wirtschaftsstörungen konjunktureller Art (Art. 198-212 CESCA-V).

Ferner wird eine gemeinsame auswärtige Wirtschaftspolitik angestrebt, wobei in einer ersten Stufe von fünf Jahren bereits die Handelspolitik zu vereinheitlichen ist (Art. 238 CESCA-V) sowie die Harmonisierung der Währungspolitiken mittels koordinierten Einsatzes des Notenbankinstrumentariums und der Ausgleichung der globalen Zahlungsbilanzungleichgewichte (Art. 247-255 CESCA-V). Auch eine gemeinsame Behandlung ausländischer Investitionen ist vorgesehen, die bereits innerhalb von sechs Monaten nach Inkrafttreten des Vertrags vom Rat zu verabschieden ist (Art. 256-259 CESCA-V). Das geschilderte Integrationsbild erhält seine Abrundung durch gemeinsame Bestimmungen über Wissenschafts-. und Technologiepolitik (Art. 260-262 CESCA-V) sowie über Infrastruktur-, Verkehrs- und Energiepolitik (Art. 260-276 CESCA-V). Neben dem eben beschriebenen, weit gespannten Integrationsansatz, der - bei geringstmöglicher Festlegung von Fristen - doch spätestens innerhalb von 25 Jahren nach Inkrafttreten des Vertrages zu vollenden wäre ${ }^{61}$, verdient aber auch die Organstruktur besonderes Interesse, da sie die bisherigen institutionellen Errungenschaften der zentralamerikanischen Integrationsversuche überträfe. Dies verwundert nicht, da die CESCA

\footnotetext{
$57 \mathrm{Vgl}$. Art. 149, 160 CESCA-V: „Salubridad, ordén público, seguridad o interés nacional“; vgl. aber auch Art. 36, 48 III, 56 EWG-V.

58 "Agreement on the System of Central American Integrated Industries“ vom 10. 6. 1958 mit Anderungsprotokollen sowie "Central American Agreement on Fiscal Incentives to Industrial Development ${ }^{\alpha}$ vom 31.7. 1962 mit Anderungsprotokoll in: Intal, Instruments II, a. a. O. (Anm. 29),. S. 451 ff.; hierzu 31. 7. 1962 mit Anderungsprotokoll in:
Peterwerth, a. a. O. (Anm. 37), S. $12 \mathrm{f}$.

59 Art. 214-224, 225-227 CESCA-V.

60 S. auch Informe, a. a. O. (Anm. 34), S. 57.

61 Art. 1 Abs. 2 CESCA-V.
} 
ihr institutionelles Vorbild der EG entnimmt, die auf die Integrationsbemühungen der lateinamerikanischen Staaten immer wieder stimulierend und befruchtend einwirkt ${ }^{62}$.

Als Hauptorgane der Gemeinschaft sind der „Rat“ (Consejo), die „Kommission“ (Comisión) sowie der "Gerichtshof" (Tribunal) projektiert ${ }^{63}$. Daneben sollen als spezielle Organe der bereits existierende "Zentralamerikanische Währungsrat"64 und die "Zentralamerikanische Bank für wirtschaftliche Integration"65 fungieren. Eine Reihe namentlich genannter oder noch zu gründender technisch-administrativer Institutionen vervollständigt das Organbild. Schließlich bleibt der dem „Wirtschafts- und Sozialausschuß" der EWG nachgebildete Ausschuß gleichen Namens und gleicher Funktion ${ }^{66}$ zu erwähnen. Eine nähere Beschreibung der Hauptorgane, die in ihrer Zuordnung ungefähr der Funktionenteilung der EG-Organe entsprechen, kann hier unterbleiben. Es erscheint schon lohnender, die auffälligsten Unterschiede zu den entsprechenden EG-Organen hervorzuheben ${ }^{67}$. Die Aufgaben von "Rat" und „Kommission" sind detaillierter als im EWG-V niedergelegt ${ }^{68}$; auch für die CESCA dürfte das „Prinzip der begrenzten Einzelermächtigung“ gelten, wonach die Organe nur aufgrund ausdrücklicher vertraglicher Ermächtigung tätig werden können ${ }^{69}$. Dementsprechend enthält der Vertrag Handlungsermächtigungen in allen Wirtschaftsbereichen bereit, die zur Ausübung eines Vorschlags der Kommission bedürfen ${ }^{70}$. Den Abstimmungserfahrungen der EWG wie den Souveränitätsvorstellungen der Mitglieder Rechnung tragend, ist für Abstimmungen im „Rat" grundsätzlich Einstimmigkeit erforderlich, falls der Rat nicht ad hoc etwas anderes beschließt ${ }^{71}$; eine Stimmrechtsgewichtung kennt der Entwurf nicht ${ }^{72}$. Entscheidungen der fünf Kommissionsmitglieder sind grundsätzlich konsensual vorzunehmen; nur im Falle der Nichteinigung ist einfache Mehrheitsentscheidung möglich ${ }^{73}$. Art, Erlaß und Reichweite der Organakte sind zwischen „Rat" und „Kommission“ unterschiedlich geregelt und weichen sowohl von der Normierung des EWG-Vertrags wie der bestehenden zentralamerikanischen Praxis ab. Während die Organe des MCCA, der „Zentralamerikanische Wirtschaftsrat" und der „Exekutivrat“, „Entscheidungen“ in Form von „Beschlüssen“ oder „Empfehlungen“ erlassen können ${ }^{74}$, könnte im Rahmen der CESCA nur der Rat als höchstes Gemeinschaftsorgan "Verordnungen“ (decretos) und "Gemeinschaftsbeschlüsse" (acuerdos comunitarios) verabschieden, die 20 Tage nach Veröffentlichung im Amtsblatt in Kraft treten ${ }^{75}$. Die „Verordnungen“ würden in ihrer Rechtsnatur den EWG-Verordnungen entsprechen, während die „Gemeinschaftsbeschlüsse“ Organ-

\footnotetext{
62 Dies ließe sich auch für den institutionellen Rahmen des Andenpakts belegen; s. Petersmann, a. a. O. (Anm. 2), S. 63; Orrego-Vicuña, a. a. O. (Anm. 16), S. 161.

63 Art. 9 Abs. 2 CESCA-V. Schon die Bezeichnung der Organe stimmt mit jener der EG überein.

64 "Consejo Monetario Centroamericano ; Art. IV Abkommen über die Währungsunion (Anm. 46)

65 "Agreement Establishing the Central American Bank for Economic Integration ${ }^{*}$ vom 13. 12. 1960 in Managua, in: Intal, Instruments, a. a. O. (Anm. 29), S. $505 \mathrm{ff}$.

66 „Comité Económico y Social“ (Art. 42-45 CESCA-V).

67 Dieser Vergleich wäre - bei entsprechender Realisierung der CESCA - eine eigene Studie wert.

68 Rat: Art. 13a - 11; Kommission: Art. 26a - n. 69 Hierzu Bleckmann, Europarecht (1976), S. 46 ff.; Ipsen, a. a. O. (Anm. 39), S. 425 ff.

70 Vgl. Art. 20 CESCA-V.

71 Art. 18, 19 CESCA-V. Kommissionsvorschläge können aber - ähnlich Art. 149 EWG-V - nur einstimmig zurückgewiesen werden (Art. 20 Abs. 2 CESCA-V).

$72 \mathrm{Vgl}$. demgegenúber die Stimmenwägung bei qualifizierten Mehrheitsentscheidungen nach Art. 148 EWG-V. $\mathrm{Zu}$ den Abstimmungsmodalitäten des CESCA-Entwurfs s. die SIECA-Studie, a. a. O. (Anm. 56), S. 40 ff.

73 Art. 38 CESCA-V; Art. 17 FusV-EG.

74 Die Beschlüsse ("resoluciones") umfassen sowohl den Erlaß von Verordnungen, verbindliche Entscheidungen, interne Richtlinien und Vertragsänderungen. Zu den Handlungsformen s. Intal, a. a. O. (Anm. 16), S. $680 \mathrm{ff}$; S Schulz, a. a. O. (Anm. 29), S. $78 \mathrm{ff}$.

75 Vgl. Art. 46, 47 CESCA-V.
} 
akte enthielten, die nicht allgemein verbindlichè Geltung besäßen ${ }^{76}$. Demgegenüber könnte die „Kommission“ nur „Beschlüsse“ (resoluciones) fassen, die sowohl für Staaten wie für Individuen bindend wären, Vorschläge dem Rat unterbreiten sowie Empfehlungen und Weisungen abgeben ${ }^{77}$.

Aus dieser unterschiedlichen Ausstattung und Reichweite der Organakte wird das souveränitätsorientierte Integrationsverständnis Zentralamerikas deutlich, das nur dem Rat den Erlaß allgemein verbindlicher Normen vorbehält ${ }^{78}$. Zeigt schon ein Vergleich der Organakte erhebliche Nachteile gegenüber dem EWG-Vertrag, so gilt dies erst recht für den Geltungsvorrang des Gemeinschaftsrechts, ein andauerndes Kernproblem des mittelamerikanischen Integrationsrechtes. Das herkömmliche "Gemeinschaftsrecht" des MCCA ließ sich schon bisher nicht dem nationalen Verfassungsrecht überordnen ${ }^{79}$, und selbst für Normen des Sekundärrechts waren häufig Transformationsakte erforderlich ${ }^{80}$. Der CESCA-Entwurf scheint hier keinen wesentlichen Fortschritt zu bringen, da er den Vorrang des Gemeinschaftsrechts nicht eindeutig sicherstellt, sondern im Falle sekundärer Rechtsnormen den Vorrang gegenüber nationalem Recht von der ausdrücklichen Bestimmung im Organakt abhängig macht - eine schon für den MCCA gebräuchliche Praxis ${ }^{81}$ - und im Falle der Unvereinbarkeit einer solchen Norm mit nationalem Verfassungsrecht eine Beratung und Abstimmung mit den zuständigen nationalen Verfassungsorganen auferlegt ${ }^{82}$; damit wird dem nationalen Verfassungsrecht Vorrang vor sekundärem Gemeinschaftsrecht eingeräumt. Selbst der Vorrang des primären Gemeinschaftsrechts vor nationalem Verfassungsrecht ist nicht eindeutig beantwortet. Die Möglichkeit eines solchen Normenkonflikts wird mit der Begründung geleugnet, daß die Staaten mit der Ratifikation des Vertrages die Vereinbarkeit mit ihrem jeweiligen Verfassungsrecht zu erkennen gäben und überdies die „pacta-sunt-servanda“-Regel die Priorität des Vertragsrechts sichere ${ }^{83}$. Eine dem Europäischen Gerichtshof vergleichbare Rolle spielt der projektierte Gerichtshof der CESCA, dem die Wahrung des Rechts bei Auslegung und Anwendung des Vertrags aufgetragen ist (Art. 50 CESCA-V). Die Möglichkeit von Nichtigkeitsklagen nach Art. 60 a, 68, 69 CESCA-V ist Art. 173 EWG-V, die Untätigkeitsklage nach Art. 65 CESCA-V dem Art. 175 EWG-V, die Klage der Kommission auf Feststellung einer Vertragsverletzung durch einen Mitgliedstaat nach Art. 72 CESCA-V dem Art. 169 EWG-V, die Klage eines Mitgliedstaats gegen einen anderen Mitgliedstaat nach Art. 73 CESCA-V dem Art. 170 EWG-V und die Möglichkeit der Vorabentscheidung nach Art. 63 CESCA-V dem Art. 177 EWG-V nachgebildet. Neben weiteren Zuständigkeiten. (z. B. bei Schiedsklauseln, Schadensersatzforderungen, Streitigkeiten zwischen Mitgliedern) ließe der Entwurf auch eine Kompetenzerweiterung durch einstimmigen Ratsbeschluß zu (Art. 66 CESCAV). Ob die Nachahmung der EuGH-Kompetenzen, insbesondere das vorgesehene Vorabentscheidungsverfahren, die souveränitätsbewußte Einstellung der mittelame-

76 S. die SIECA-Studie, a. a. O. (Anm. 56), S. 61.

77 Art. 46 Abs. 4 CESCA-V; SIECA-Studie, a. a. O. (Anm. 56), S. 61 f.

78 SIECA-Studie (Anm. 56), S. 60.

79 Vgl. Schulz, a. a. O. (Anm. 29), S. $100 \mathrm{ff}$

80 Schulz, ebd., S. 92

81 S. Orrego-Vicuña, a. a. O. (Anm. 16), S. 148; Intal, a. a. O. (Anm. 16), S. 995 ff.

82 Vgl. Art. 48 CESCA-V; Informe, a. a. O. (Anm. 34), S. 54 . pacta-sunt-servanda-Regel kann aber einen Normenkonflikt nicht ausschließen; ein Staat kann etwa nach einer vollzogenen Verfassungsänderung aus verfassungsrechtlichen Gründen nicht mehr in der Lage sein, gewisse völkervertragliche Pflichten zu erfüllen; dies stellt ihn vor die Alternative der völkerrechtlichen Haftung oder des Verfassungsbruchs. 
rikanischen Staaten gegenüber supranationalen Integrationsbestrebungen einzudämmen imstande wäre, müßte die Praxis erweisen. Immerhin scheint man angesichts der positiven Erfahrungen mit dem EuGH als „Integrationsfaktor" seine Hoffnungen ebenfalls in einen judiziell verstärkten Integrationsprozeß zu setzen, eine Perspektive, die auch im Rahmen des Andenpakts durch die Vorlage eines ebenfalls am EuGH-Modell ausgerichteten Andengerichtshofstatuts erkannt wurde ${ }^{84}$.

Während der CESCA-Entwurf für Zentralamerika einen integrationspolitischen Durchbruch bedeuten würde - sofern das Projekt in der vorliegenden Fassung überhaupt angenommen wird - , dürfte er doch im gesamtlateinamerikanischen Integrationszusammenhang vom Andenpakt noch übertroffen werden.

\section{Der Andenpakt}

Der am 26. Mai 1969 zwischen Bolivien, Chile, Ekuador, Kolumbien und Peru geschlossene Andenpakt (Pacto Andino = PA) stellt einen subregionalen Präferenzraum innerhalb der 1960 gegründeten Lateinamerikanischen Freihandelszone (ALALC) dar ${ }^{85}$. Der Andenpakt ist die bisher wohl erfolgreichste Wirtschaftsgruppierung in Lateinamerika ${ }^{36}$ und übertrifft in seiner "supranationalen“ Ausrichtung die bis dato geschaffenen Integrationsgebilde bei weitem; in dieser Hinsicht dürfte er sogar den ehrgeizigen Integrationsentwurf der CESCA noch in den Schatten stellen. Entscheidende Vorteile des Andenpakts sind - bei vergleichbarer institutioneller Struktur ${ }^{87}$ - die Möglichkeit von Mehrheitsabstimmungen der als Staatenvertretungsorgan fungierenden „Kommission“ (Comisión) ${ }^{88}$ sowie die grundsätzliche Anerkennung des Geltungsvorrangs des gemeinschaftlichen Primärund Sekundärrechts vom nationalen (auch Verfassungs-)Recht ${ }^{89}$ und die Möglichkeit einer Durchgriffswirkung des von den Organen gesetzten Sekundärrechts ${ }^{90}$. Würde man die Elle der „Supranationalität“ überhaupt auf lateinamerikanische Integrationsverbände anlegen, so fände dies beim Andenpakt eine gewisse Berechtigung'.

Der Andenpaktvertrag erfuhr durch den Beitritt Venezuelas im „Konsens von Lima" vom 13. 12. 1973 bereits gewisse Modifikationen ${ }^{92}$; durch den am 30. 10. 1976 erfolgten Austritt Chiles aus dem Andenpakt sind wichtige Änderungen zu verzeichnen, die hier ein kurzes Resümee verdienen.

Der Austritt Chiles wurde durch die unüberbrückbaren Meinungsverschiedenheiten mit seinen Partnern vor allem in der Frage der Festlegung des gemeinsamen Außenzolls wie einer liberalen Einstellung zu ausländischen Investitionen ausgelöst ${ }^{93}$. Für Chile erloschen sämtliche Mitgliedsrechte und -pflichten einschließlich

\footnotetext{
84 Hierzu Orrego-Vicuña, La création d'une cour de justice dans le groupe andin, Cahiers de Droit Européen (1974), S. $135 \mathrm{ff}$. Der Gerichtshof sollte noch 1977 errichtet werden.

85 Text in ILM 8 (1969), S. $910 \mathrm{ff}$.

86 Hierzu Hummer, a. a. O. (Anm. 1), S. $80 \mathrm{ff}$; Petersmann, a. a. O. (Anm. 2), S. $62 \mathrm{ff} ., 177 \mathrm{ff} ., 200 \mathrm{ff}$

87 Petersmann, ebd., S. 66; Hummer, ebd., S. 92.

88 S. Art. 11 PA mit den Ausnahmen der Annexe I-III.

89 Orrego-Vicuña, a. a. O. (Anm. 16), S. 462.

90 Villagrán-Kramer, Sistematización del la estructura jurídica del Acuerdo de Cartagena, Derecho de Integración 12 (1973), S. 31; ebenso Garcia-Amador, Naturaleza y validez de los actos de los órganos subregionales andinos, Derecho de la Integración 9 (1976), S. $17 \mathrm{f}$.

91 Orrego-Vicuña, a. a. O. (Anm. 16), S. 119: $n$.. Subregional Agreement ... with accentuated characters of supranational organization.....

92 Text in Intal, Instruments, I, a. a. O. (Anm. 29), S. 219 ff. Avery: Oil, Politics and Economic Policy Making. Venezuela and the Andean Common Market; International Organization 30 (1976), S. $541 \mathrm{ff}$.

93 Chile verlangte die Freigabe der Gewinntransfers ausländischer Unternehmen sowie eine Herabsetzung der Außenzölle auf 10-30 Prozent; vgl. NZZ vom 2. 9. 1976 und 10. 9. 1976.
} 
der Zolliberalisierungskonzessionen ${ }^{94}$; allerdings bleiben die Regelungen der Entscheidung Nr. 46 über das „Gemeinsame Regime für multinationale Gesellschaften und die Behandlung subregionalen Kapitals" auch für Chile weiter in Kraft ${ }^{95}$. Durch das Lima-Protokoll vom 30. 10. $1976^{96}$ wurden die wichtigsten vertraglichen Fristen um drei Jahre verlängert. Die Liberalisierung des Binnenhandels durch automatische und unwiderrufliche Zollsenkungsschritte muß nun bis 1983 vollzogen sein; dies gilt für die Produkte der sektoralen industriellen Entwicklungsprogramme wie für die in einer eigenen Liste zum Vertrag enthaltenen Produkte, die 55 Prozent der gesamten Zollnomenklatur NABANDINA umfassen ${ }^{97}$. Die auf Ausnahmelisten figurierenden und von der automatischen Zollsenkung ausgenommenen Produkte sind bis $1978 \mathrm{zu}$ liberalisieren ${ }^{98}$. Bis Ende 1983 ist auch der gemeinsame Außenzolltarif durchzuführen; ab 1980 werden sich die Mitglieder einem von der Junta erarbeiteten und von der Kommission verabschiedeten gemeinsamen Zolltarif in jährlichen, automatischen und linearen Zollsenkungsschritten annähern; für Bolivien und Ekuador sind als "less developed countries" verlängerte Fristen eingeräumt ${ }^{99}$. Im Rahmen der sektoralen Entwicklungsprogramme, einem fundamentalen Integrationsinstrument zur Industrieförderung, ist der Außenzoll weiterhin nach Maßgabe des jeweiligen Programms zu entrichten ${ }^{100}$.

Bemerkenswert sind vor allem die Änderungen im Rahmen des "Lima-Statuts“, das die Regelungen über die Behandlung des Auslandskapitals enthält und seit Juli 1971 in Kraft ist ${ }^{101}$. Die bislang wohl einschneidendsten Regelungen der Behandlung ausländischen Kapitals und ausländischer Technologie durch Integrationsverbände von Entwicklungsländern ${ }^{102}$ haben durch die Entscheidungen Nr. 103 und 109 der Kommission eine Milderung erfahren, die das Investitionsklima im Andenpaktraum verbessern dürften ${ }^{103}$. Wenn auch Chile mit seinen Forderungen nach einer grundlegenden Revision der seiner Ansicht nach zu starren Bestimmungen des "Lima-Statuts" nicht durchdringen konnte, haben die Reststaaten des Andenpakts einzelne Regelungen zumindest flexibilisiert, wobei das Grundkonzept freilich erhalten blieb.

Eine Genehmigung der Beteiligung ausländischer Investoren an „nationalen“ oder "gemischten" Unternehmen ${ }^{104}$ ist künftig auch möglich, wenn die Beteiligung wie bisher - über eine Kapitalerhöhung erfolgt und wenigstens der Charakter eines gemischten Unternehmens (d. h. mindestens 51 Prozent inländische Kapitalbeteiligung) erhalten bleibt ${ }^{105}$. Der Satz für genehmigungsfreie Reinvestitionen aus dem Reingewinn eines ausländischen Unternehmens wurde von fünf Prozent auf

94 S. Decision No. 102 vom 30. 10. 1976, Integración Latinoamericana (1976), Nr. 9, S. 56.

95 „Uniform Regime on Multinational Enterprises and Regulations of the Treatment Applicable to Subregional Capital“ vom 19. 12. 1971 mit Änderung vom 13. 2. 1973, Intal, Instruments, I, a. a. O. (Anm. 29), S. $326 \mathrm{ff}$; hierzu Petersmann, a. a. O. (Anm. 2), S. $200 \mathrm{ff}$.

96 "Lima Protocol Additional to the Cartagena Agreement“ vom 30. 10. 1976, in: ILM XVI (1977), S. $236 \mathrm{ff}$. Das Protokoll wurde inzwischen in den Andenpakt integriert: Acuerdo de Cartagena Texto Oficial Codificado (Entscheidung No. 117 der Kommission), Integración Latinoamericana (1977) No. 12, S. $63 \mathrm{ff}$.

97 Vgl. Art. 45a, d PA, Art. 1 Lima-Protokoll.

98 Art. 55 Abs. 3 PA, Art. 1 Lima-Protokoll.

99 Bis 1988: Art. 62 Ábs. 3, 104 PA; Art. 1 Lima-Protokoll.

100 Art. 65a PA.

101 Decision No. 24 vom 31. 12. 1970 (ergänzt durch Decision No. 37, 37-A und 70): Common Regime of Treatment of Foreign Capital and of Trademarks, Patents, and Licenses, and Royalties; Intal, Instruments, I (Anm. 29), S. $296 \mathrm{ff}$.; Literaturnachweise bei Hummer, a. a. O. (Anm. 2), S. 95.

102 Petersmann, a. a. O. (Anm. 2), S. $177 \mathrm{f}$.

103 Andean Commission: Codified Text of the Andean Foreign Investment Code, as of November 30, 1976;

104 Die Definitionen des Art. 1 der Decisión No. 24 bleiben im wesentlichen unverändert. Als nationale Unternehmen gelten solche mit mindestens 80 Prozent inländischer, als gemischte Unternehmen solche zwischen 51 und 80 Prozent inländischer Kapitalbeteiligung; ausländische Unternehmen sind solche, die mehr als 49 Prozent des Kapitalanteils innehaben.

105 Art. 4 Lima-Statut. 
sieben Prozent erhöht ${ }^{106}$. Der kurz- und mittelfristige lokale Kapitalmarkt ist für ausländische Unternehmen nach Maßgabe der jeweiligen nationalen Gesetzgebung geöffnet und nicht mehr von gemeinschaftsrechtlichen Regelungen abhängig; der Zugang zu langfristigem Kapital des Sitzstaats bleibt verwehrt ${ }^{107}$. Die in Art. 28 Lima-Statut niedergelegten Fristen für die Umwandlung bestehender ausländischer Firmen in nationale oder gemischte Unternehmen laufen nunmehr ab 1. Januar 1974; ausländische Firmen in Kolumbien, Peru und Venezuela müssen sich bis Ende 1988, in Bolivien und Ekuador bis Ende 1993 umwandeln, falls sie für ihre Produkte die Zolliberalisierung des Andenpakts in Anspruch nehmen wollen. Die - innerhalb drei Jahren nach Inkrafttreten des Lima-Statuts zu deklarierende ${ }^{\mathbf{1 0 8}}-\mathrm{Um}$ wandlung kann nunmehr auch im Wege einer Kapitalerhöhung erfolgen ${ }^{109}$. Der Satz des jährlichen Gewinntransfers des Wertes der registrierten ausländischen Investition wird von 14 Prozent auf 20 Prozent angehoben, wobei die Mitglieder jedoch auch höhere Transfers genehmigen können ${ }^{110}$. Ausländische Unternehmen, die mehr als 80 Prozent in Drittländer exportieren, waren von diesen Umwandlungs- und Transferregelungen ausgenommen; dies gilt nun auch für ausländische und gemischte Unternehmen des Tourismus ${ }^{111}$. Schließlich werden Investitionen „internationaler Finanzorganisationen “ und „fremdstaatlicher Entwicklungsorganisationen" als "neutrales" Kapital behandelt und bei der Berechnung der Kapitalanteile ausgeklammert ${ }^{112}$. Gerade diese Möglichkeit „neutraler Investitionen“ ausländischer Staaten im Verbund mit ausländischen Firmen dürfte die Härten des Umwandlungsrechts mildern helfen ${ }^{113}$.

$\mathrm{Ob}$ der durch den vollzogenen Austritt Chiles in eine schwere Krise geratene Andenpakt sich durch die vorgeschlagenen Änderungen revitalisieren kann, bleibt vorerst abzuwarten. Immerhin erscheint es nicht ausgeschlossen, daß der Andenmarkt trotz räumlicher Verkleinerung durch die größere Homogenität der wirtschaftspolitischen Auffassungen seiner Mitglieder und der Liberalisierung seiner Investitionspraxis neuen Auftrieb erhält.

\section{Die Lateinamerikanische Freihandelszone (ALALC)}

Im Gegensatz zum Andenpakt findet sich der Integrationsprozeß der ALALC noch immer in der Stagnation. Dies dürfte letztlich in der unterschiedlichen Wirtschaftskraft zwischen den großen Ländern Argentinien, Brasilien und Mexiko, den Ländern „mit unzureichenden Binnenmärkten“ (Kolumbien, Chile, Peru und Venezuela) und den „weniger entwickelten Ländern“ (Bolivien, Ekuador, Paraguay und Uruguay) begründet sein ${ }^{114}$. Diese Disparität zeigte sich etwa in ungleichgewichtigen Handelsströmen zwischen den Partnern, hohen Protektionsraten zugunsten inländischer Unternehmen und einer fehlenden Koordinierung der wirtschaftsund handelspolitischen Instrumente ${ }^{115}$. Die Unfähigkeit, die vertraglich vorgesehene

106 Art. 13 Lima-Statut.

107 Art. 17 Lima-Statut.

108 Art. 28, Art. A der Temporary Provisions, Lima-Statut.

109 Art. 31 Abs. 2 Lima-Statut.

110 Art. 37 Abs. 1 und 2 Lima-Statut.

111 Art. 34 Abs. 2 Lima-Statut.

112 Noch unbezifferter Artikel im Anhang des Lima-Statuts.

113 Vgl. auch Handelsblatt vom 16. 12. 1976, S. 21.

114 Hummer, a. a. O. (Anm. 1), S. 74; Petersmann, a. a. O. (Anm. 2), S. 61. 
Übergangsfrist von zwölf Jahren für die interne Zolliberalisierung einzuhalten, bestätigte bereits das Protokoll von Caracas vom 12. Dezember 1969, das die Anpassungsfrist bis Ende 1980 verlängerte und den Mindestliberalisierungssatz herabsetzte116. Die Aushandlung der auf die "nationalen Listen“ oder die „gemeinsame Liste“ zu setzenden Zollkonzessionen ist nach ersten Anfangserfolgen ins Stocken geraten; die meisten Konzessionen werden nun beim Abschluß von „Komplementaritätsabkommen" gewährt, die der Förderung der industriellen Sektoren dienen und von denen bisher 21 in Kraft sind ${ }^{117}$. Als wichtigstes Ereignis im ALALC-Integrationsprozeß der letzten Jahre ist wohl das Inkrafttreten des Protokolls vom 12. 12. $1966 \mathrm{zu}$ vermerken, das den "Rat der Außenminister" zum höchsten Organ des Verbandes bestellte; bis dahin fungierte der „Rat“ als Spezialkomitee der „Konferenz der Vertragsparteien“118. Die „Konferenz der Vertragsparteien" bleibt aber weiterhin für die mühselige Aushandlung der Zollkonzessionen zuständig, die in den Jahren 1974-1976 kaum Fortschritte erbrachte ${ }^{119}$. Ob bis 1980 noch der entscheidende Durchbruch zur Vollendung des Binnenzollabbaus gelingt, erscheint daher angesichts des gegenwärtigen Integrationsstands weiterhin fraglich ${ }^{120}$.

\footnotetext{
115 Heldt, Schwierigkeiten und Umstrukturierungstendenzen in der Lateinamerikanischen Freihandelszone, EA (1975) Nr. 1, S. 340.

116 Protocol amending the Montevideo Treaty (Resolution 261), in: Intal, Instruments, I, (Anm. 29), S. 26 ff.

117 Integración Latinoamericana (1976), Nr. 10, S. 47. Ausführlich Hummer, a. a. O. (Anm. 2), S. 52 ff.

118 Das Protokoll trat am 27. 9. 1975 in Kraft, s. Comercio Exterior 9 (1975), S. 981 .

$119 \mathrm{Heldt}$, a. a. O. (Anm. 115), S. $341 \mathrm{ff}$.; Integración Latinoamericana (1977), Nr. 12, S. 47.

120 Zum Entwicklungsstand der ALALC vgl. auch die Dokumentation der Intal, El proceso de integración en América Latina en 1976, informe anual, Buenos Aires, BID/Intal, 1977.
} 